\title{
Evaluating the Project Coordination Performance Using Information Centrality
}

\author{
Pelin Ipek TIMURCAN ${ }^{1}$ and Sevgi Zeynep DOGAN ${ }^{2}$
}

${ }^{1}$ Department of Architecture, Izmir Institute of Technology, 35430, Gulbahce, Urla, Izmir, Turkey; PH (+90) (232) 750-7054; FAX (+90) (232) 750-6000; email: pelintimurcan@iyte.edu.tr

2 Department of Architecture, Izmir Institute of Technology, 35430, Gulbahce, Urla, Izmir, Turkey; PH (+90) (232) 750-7040; FAX (+90) (232) 750-6000; email: sevgidogan@iyte.edu.tr

\begin{abstract}
Evaluation of coordination performance in a project network requires reliable measures and monitoring methods for effective management. Recent literature includes studies addressing the relationship between coordinative activity and the configuration of communication networks. In these works, the role of network centrality is investigated through the basic standard centrality measures of degree, betweenness and closeness. Current social network analysis research emphasizes new formulations of centrality measures for robust structural analysis of project networks.

This paper presents a novel approach for measuring network centrality using the concept of information centrality. It is based on the idea that all paths carry information. The significance of information centrality values for the actors in a wayfinding signage project at Istanbul Sabiha Gokcen International Airport is investigated. A centrality index is defined for each firm based on the average of the four measures of centrality. Findings suggest the existence of a high correlation between coordination scores and the centrality indices. A centrality index augmented by information centrality measure has potentials for assessing the coordination performance in construction management research, and it is promising for the structural analysis of project communication networks.
\end{abstract}

\section{INTRODUCTION}

Communication networks and the information exchange play a critical role in the coordinative activity of project participants (Hossain et al. 2006; Hossain 2009). The social network characteristic of centrality has the potential of identifying project participants in coordinator roles. Traditionally, contractual links within the project participant network define the coordinator role but recent findings argue that a central position in the communication network of a project organization may also be an indication of a party's coordinator role (Dogan et al. 2012; Dogan et al. 2013; Hossain et al. 2006; Hossain 2009). The correlation between network centrality and coordination is widely discussed in social studies (Bavelas 1950; Freeman 1978/79; Leavitt 1951). However, the construction research domain lacks such discussion apart from the work of Hossain et al. (2006), Hossain (2009) and Dogan et al. (2012). Recently, Dogan et al. (2013) proposed an uncomplicated, quantitative method to predict a firm's coordination score via a centrality index based on social network analysis. In this recent study, three standard centrality measures of the firms in a wayfinding signage project at a major airport construction were calculated and a centrality index was defined for 
each firm using the average of these centrality measures. Findings showed that the coordination scores of the firms are highly correlated with the centrality indices.

Centrality measures the distribution of relationships and describes the influence of a node based on how well connected the node is in a network. Three basic measures, degree, betweenness and closeness are proposed by sociologists to determine the centrality of a node in a social network (Freeman 1978/79). Different measures of centrality capture different aspects of a node according to its role within the network. As Freeman argued in his seminal paper (1978/79) that degree of centrality indexes a node's activity, whereas betweenness centrality measures a node's control, and closeness centrality measures its communication efficiency. Previous empirical studies provide nodes that exhibit high centrality and thus achieve high levels of performance (Ahuja et al. 2003; Pryke 2004; Schilling and Phelps 2007; Tallberg 2004; Wasserman and Faust 1994). However, over the years, researchers have proposed different measures, such as information centrality (Stephenson and Zelen 1989) focusing on different features of central nodes.

Our purpose in this paper is to introduce a novel approach for evaluating network centrality based on the concept of information centrality that has been overlooked for the analysis of communication networks in construction research. The measure of information centrality weighs all paths between a pair of nodes assuming all paths carry information and quantifies the relevance of each of the nodes in the network. It has a rationale and provides readily interpretable data. The calculations are relatively straightforward and can be done for large networks. The use of quantifiable values of information centrality can also illustrate communication structures, patterns and relationships in an e-mail network (Stephenson and Zelen 1989).

The paper is organized as follows: Descriptions and formulas for information centrality are presented in detail followed by the description of the construction project case study. The methodology part includes the calculations for information centrality measures for each of the firms in the construction project and a centrality index based on the average of all four centrality measures is defined. Finally, the correlation between coordination performance and network centrality are evaluated, findings are discussed and concluding remarks are made.

\section{INFORMATION CENTRALITY}

The concept of information centrality is essential for the proposed approach in this paper. As an earlier reference for centrality measures, Freeman's (Freeman 1978/79) betweenness centrality encompasses the betweenness counts focusing only on geodesics of the given network paths. This leads to the neglect of the paths with distances greater than the minimum path length attained by the geodesics. In communication relations, actors might choose message paths that are longer than the geodesics under the influence of strong reliability reasons or intrinsic system characteristics. Information might be deliberately channeled through various communication paths created via many intermediaries (Stephenson and Zelen 1989). In these form of networked communications, information robustness in a network with its all-possible paths is vital where information might take an indirect route.

The index of centrality concept developed by Stephenson and Zelen (1989) comprises all the paths between network actors by assigning a weight coefficient to the each path depending on its length. In this framework, a weighted function of 
the paths is calculated using the inverses of the lengths of the paths as weights. Geodesics are given as weights of unity, while longer paths than the geodesic length receive smaller weights based on the contained information (Wasserman and Faust 1994). The information of a path is defined quite simply as the inverse of its length.

The concept of information is used extensively both in the communication and the statistical estimation theory. Information is statistically defined as the inverse of the variance of an estimator. If an estimator has a small variance, it has large information which is considered positive. The opposite case is also a valid argument where poor estimators with large variances have little information. This approach is applied to the network centrality framework by extending betweenness on geodesics to all possible paths and weighting according to the information contained by these particular paths (Stephenson and Zelen 1989).

Stephenson and Zelen (1989) argued that the information centrality of an actor is a function of all the information for the paths flowing out from that specific actor. The chosen function is the harmonic average. The procedural arguments given below presents Stephenson and Zelen's (1989) approach in mathematical details:

- If $\left(t_{i}\right)$ refers to a pair of nodes in the graph, the first phase is the identification of all possible paths connecting $i$ and $j$. Suppose that there are $k_{i j}$ paths connecting $i$ and $j$; accordingly the existing paths for $(i, D)$ are

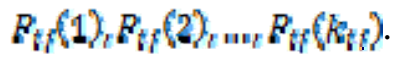

- Then topological distance $\left(d_{*}\right)$ is defined for all the denoted paths, which is the sum of the number of existing links on the specified path. Distance

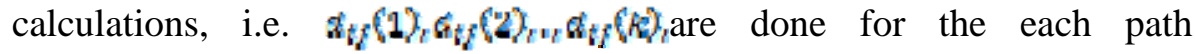

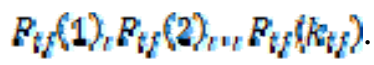

- The information measure between two nodes $i$ and $j\left(l_{i j}\right)$ is defined as the reciprocal of the topological distance $i d_{i j}$ between them: $t_{i j}=1 / d_{i j}$. The information of node $(t)$ is the sum of all information content with regard to

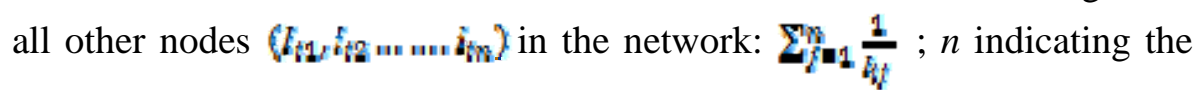
total number of nodes in the network.

- The centrality information measure of node $(i)$ is defined by the harmonic average of the information flowing from the the all other nodes in the network:

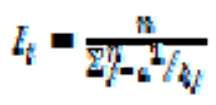

\section{CASE STUDY}

This study re-evaluates the correspondence of coordination scores and network centrality measures of the project participants of the Istanbul Sabiha Gökçen International Airport (ISGIA) wayfinding signage project by using information centrality concept. Prior to this study, the interplay between coordinative activity and network centrality in the project was initially studied by using degree, betweenness and closeness measures and the findings were reported (Dogan et al. 2013). 
The wayfinding signage design project of the ISGIA's new terminal building's interiors and exteriors is used as a case study in this paper. Being one of the largest capital investment project in Turkey, ISGIA is a major airport located on the Asian part of Istanbul and ISGIA project was undertaken by using buildoperate-transfer (BOT). The owner of the project was Airport Management and Aviation, Inc. and the other party in the BOT arrangement was the Limak-GMRMAHB consortium. The consortium awarded the architectural design project through a design competition to the Tekeli-Sisa Architectural Partnership, and the construction contract to the joint venture Limak-GMR. The joint venture LimakGMR subcontracted the wayfinding signage design/build (D/B) project to YONSIS, who used Woodhead as a consultant to perform the schematic design, developing signs, specifying materials, fabrication details, and font styles and colors. YONSIS also engaged RGB as a management consultant. RGB coordinated the activities of the participants of the wayfinding project by providing consulting services such as scheduling work, organizing meetings, monitoring RFIs, submittals and change orders, and overseeing the project closeout. Table 1 shows the roles of the project participants (Dogan et al. 2013).

Table 1. Roles of the project participants

\begin{tabular}{ll}
$\begin{array}{l}\text { Wayfinding Signage D/B } \\
\text { Project Participant Firms }\end{array}$ & Roles \\
\hline YONSIS & Subcontractor \\
RGB & Management Consultant \\
Woodhead & Design Consultant \\
LIMAK-GMR & General Contractor \\
Tekeli-Sisa & Designer \\
\hline
\end{tabular}

The main communication channel among the participating firms was the email exchange due to the geographic separation of the project parties. The general contractor (Limak-GMR-MAHB) was an international joint venture (LimakTurkey, GMR-India, MAHB-Malaysia), the designer (Tekeli-Sisa) and the consulting firm (RGB) were local project participants. The subcontractor in charge of the wayfinding signage design project (YONSIS) was located in Izmir, Turkey and the sub-subcontractor (Woodhead) was located in Adelaide, Australia. E-mail traffic took place over a period of six months. The complete e-mail communication data of 216 sent and received e-mails were reviewed and analyzed by Erbasaranoglu (2011).

The e-mail communications constituted a good source of data for measuring the network relationships and the coordination patterns during the project process. In order to analyze this kind of data more effectively, Dogan et al. (2013) proposed a simple procedure for monitoring the coordinative performance of project participants. The degree, betweenness and closeness centrality measures of the project participants are calculated using social network analysis on the email communication network between the participants. For defining the level of coordinative role within the project network, a centrality index is defined for each firm based on the average of the three standard centrality measures. The firm's 
coordination score is also calculated based on the content analysis of the sent and received e-mails between the project participants. The data analyses returned highly correlated values between the coordination scores and centrality indices. Findings suggested that the coordinative role of a firm can be identified by its particular centrality index. Referencing the proposed method in this paper, the centrality index can be measured relatively easy by using simple software and capturing only the number and direction of emails exchanged between the participants, rather than performing time-consuming content analysis of e-mail exchanges to calculate coordination scores.

\section{METHODOLOGY OF THE STUDY}

The methodology of the study involves two parts: (1) Conducting social network analysis for the email dataset obtained from the wayfinding signage design project; calculating information centrality measures; and defining centrality indices for project participants; and (2) Evaluating correlation between coordination performance and network centrality of project participants.

\section{Measuring Information Centrality}

Network centrality identification of social network analysis is used to examine the electronic mailing of firms participating in wayfinding signage design project. Information centrality measurements are calculated by UCINET, software developed by Borgatti et al. (2002). UCINET analyzed the information exchange data matrix for email communications between the participants of the project. The sent and received e-mails by each firm were added up provided that the recipient is the direct one other than the recipients of carbon copy ("CC" or "BCC" types); for CC and BCC e-mails are usually intended as passive information propagation, rather than establishing a two-way relationship (Klimt and Yang 2004).

Based on Freeman's (1978/79) definitions, degree centrality denotes the number of nodes connected to one node in particular; betweenness centrality is the extent to which a firm lies between other pairs of firms (it is the proportion of all the shortest paths (i.e., geodesic distances) between pairs of other firms that pass through the firm); closeness centrality is based on the sum of the geodesic distances from each node to all other nodes. Then, following Stephenson and Zelen (1989)'s definition information centrality is the harmonic average of all the information included in all the paths flowing from that firm.

Stephenson and Zelen (1989) stated that the information centrality calculations could be practically conducted by simply inverting an incidence matrix rather than the principal information centrality formula (Eq. 1) as previously introduced. A crucial component of this later formula is the sum of the strengths or values for the lines incident with a node (Wasserman and Faust 1994). The number of sent and received emails present in the weighted information exchange network of the wayfinding signage project implies compatibility for such computation. For a network with n nodes, $n \times n$ matrix $A=\left(a_{i j}\right)$ is defined:

$\sigma_{t i}=1+$ (sum of the welghts of all the links of node t)

and off-diagonal elements 


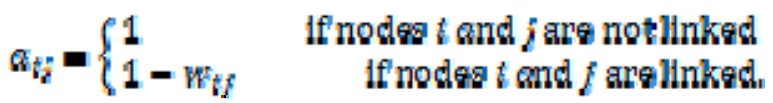

$a_{i i}$ represents the diagonal elements of matrix $A ; a_{i j}$ represents the off-diagonal elements of matrix $A ; w_{t i}$ is the weight of the link between the nodes $i$ and $j$. Then, information centrality is calculated by inverting the matrix $A$. The matrix

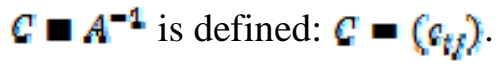

For the information measure, two intermediate quantities are required.

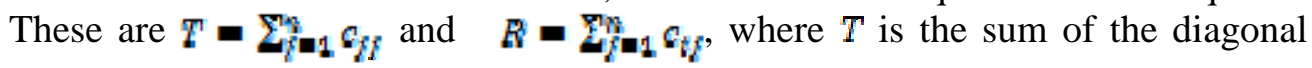
entries of the matrix, while $R$ is any one of the row sums (all the row sums are equal). With these two quantities, and the elements of $C$, finally the information centrality $I_{i}$ for node $i$ is calculated as follows:

$$
E_{t}=\frac{1}{\sigma_{t i}+\left(T-2 T_{2}\right)}
$$

Dogan et al. (2013) provided degree, betweenness and closeness centrality measures based on the email dataset used in this study. Information centrality calculations are done by UCINET for the same dataset. Information centrality formulation assumes that the relational structure is non-directional. For the directed and weighted network of the wayfinding signage project, UCINET eliminates the smaller weight indicating either the sent or the received number of emails on the adjacency matrix; and reconstructs the weighted matrix with the greater weight values assigned to the incoming or outgoing link of the node.

The results are presented in Table 2 . The centrality values were normalized so that they are independent of the size of the network, and range between 0 and 1 . The average of the three measures of centrality (degree, betweenness and closeness) in Table 2 represents the centrality index 1 of each firm (Dogan et al. 2013). The average of four measures of centrality (degree, betweenness, closeness and information) in Table 2 represents the centrality index 2 of each firm.

Table 2. Normalized Centrality Measures and Centrality Indices

\begin{tabular}{|c|c|c|c|c|c|c|}
\hline \multirow{2}{*}{$\begin{array}{l}\text { Wayfinding Signage } \\
\text { D/B Project } \\
\text { Participant Firms }\end{array}$} & \multicolumn{4}{|c|}{$\begin{array}{c}\text { Normalized Centrality } \\
\text { Measures }\end{array}$} & \multirow[t]{2}{*}{$\begin{array}{l}\text { Centralit } \\
\text { y Index } 1\end{array}$} & \multirow{2}{*}{$\begin{array}{c}\text { Centralit } \\
\text { y Index } \\
2\end{array}$} \\
\hline & Degree & $\begin{array}{l}\text { Betwee } \\
\text { n-ness }\end{array}$ & $\begin{array}{l}\text { Clos } \\
\text { e- } \\
\text { ness }\end{array}$ & $\begin{array}{l}\text { Informatio } \\
\text { n } \\
\text { Centrality }\end{array}$ & & \\
\hline YONSIS & 0.45 & 0.38 & 1.00 & 0,22 & 0,61 & 0,51 \\
\hline RGB & 0.36 & 0.13 & 0.90 & 0,21 & 0,47 & 0,40 \\
\hline Woodhead & 0.27 & 0.00 & 0.74 & 0,20 & 0,34 & 0,30 \\
\hline $\begin{array}{l}\text { LIMAK-GMR- } \\
\text { MAHB }\end{array}$ & 0.25 & 0.00 & 0.67 & 0,18 & 0,31 & 0,27 \\
\hline Tekeli-Sisa & 0.03 & 0.00 & 0.69 & 0,07 & 0,23 & 0,20 \\
\hline
\end{tabular}




\section{Evaluating the correlation between coordination performance and network centrality}

The coordination scores of the participating firms in the wayfinding signage project are provided by Dogan et al. (2012). The correlation between the coordination scores and the centrality indices was investigated by using Spearman rank correlation (Table 3). The Spearman rank order correlation coefficient $(\rho)$ is a non-parametric measure of the strength and direction of association that exists between two variables measured on an ordinal scale (Hollander and Wolfe 1999). Spearman correlation coefficient can be calculated by using the relationship presented in Eq. 5.

$$
\beta=\frac{6 \Sigma\left(x-2 z^{2}\right)}{n\left(n^{2}-1\right)}
$$

Where $x_{i}-y_{i}$ represents the differences between the ranks of the variables, and $\mathrm{n}$ is the number of variables. This coefficient takes values between -1 and +1 , where zero represents no correlation.

Table 3. Spearman Rank Correlation between Coordination Scores and Centrality Indices

\begin{tabular}{llll}
\hline $\begin{array}{l}\text { Wayfinding Signage D/B } \\
\text { Project Participant Firms }\end{array}$ & $\begin{array}{l}\text { Coordination } \\
\text { Score }\end{array}$ & $\begin{array}{l}\text { Centrality } \\
\text { Index 1 }\end{array}$ & $\begin{array}{l}\text { Centrality } \\
\text { Index 2 }\end{array}$ \\
\cline { 2 - 4 } YONSIS & 1069 & 0.61 & 0,51 \\
RGB & 1329 & 0.47 & 0,40 \\
Woodhead & 363 & 0.34 & 0,30 \\
LIMAK-GMR-MAHB & 328 & 0.31 & 0,27 \\
Tekeli-Sisa & 36 & 0.23 & 0,20 \\
\hline Spearman Rank Correlation & & $\rho=0.90$ & $\rho=0.90$ \\
Coefficient & & $\rho$ & \\
\hline
\end{tabular}

\section{FINDINGS AND DISCUSSION}

The findings of the study are discussed from two related viewpoints regarding the correlation scores. The first part evaluates the correlation between the calculated centrality indices (Centrality Index 1 and Centrality Index 2) and coordination scores and the second part focuses on the correlation of the information centrality indices with the coordination scores, in comparison to the three-standard centrality measures.

\section{Coordination vs. Network Centrality}

The calculated coordination scores and the proposed centrality index were analyzed for the possible existence of a correlation based on the information centrality measures. The immediate results from the statistical analyses suggest that coordination scores are highly correlated with the Centrality Index 2 with a Spearman rank correlation coefficient $\$ R$ ) of 0.90 (Table 3). These results support the previous claims that the network centrality has a direct statistical influence on 
coordination levels among the participants of a project network (Bavelas 1950; Dogan et al. 2013; Freeman 1978/79; Hossain et al. 2006; Hossain 2009; Leavitt 1951). The correlation between coefficient of the Centrality Index 2 and the coordination scores was as high as the correlation coefficient of the Centrality Index 1 (averaging three measures of centrality) defined by Dogan et al. (2013).

\section{Information Centrality}

Coordination scores were also checked for possible correlations against measures for each centrality type. The results show that the coordination scores are also highly correlated with centrality measures of degree, betweenness, closeness, and information. The Spearman rank correlation coefficients are given as $(\beta)$ of $0.90,0.80,0.80,0.90$ respectively. From a general perspective, it can be concluded that the perfect correlation between coordination scores and centrality measures could be achieved in case of the highest coordinative activityperforming firm RGB (management consultant) had the highest centrality index (Table 3). This particular case requires the second-ranked RGB to move to the first place instead of the highest-ranked firm YONSIS (subcontractor) in the communication network in terms of the centrality scores. Information centrality measure has the most proximate values for YONSIS and RGB $(0.22,0.21$ respectively), compared to other centrality measures of degree $(0.45,0.36)$, betweenness $(0.38,0.13)$ and closeness $(1.00,0.90)$.

Freeman (1979/80) states that betweenness and closeness based measures of point centrality are determined by the same structural elements of a communication network. Since both are the functions of the local pair dependency, all measures have in common the same structural element: the geodesic pathway. If one assumes that communication only occurs along the shortest possible path, then communication channels are by default the geodesics. This has always been a fundamental assumption in his development of betweenness, closeness and pair dependence. This theoretical stance neglects measuring communication occurring along reachable, non-geodetic pathways (Stephenson and Zelen 1989). Freeman's degree centrality indices have also a limited ability to distinguish among actors with differing centrality. The range of possible values for a degree-based index is quite small, so that such indices are not very sensitive either (Wasserman and Faust 1994). Information indices are much more inclusive than those based on betweenness, closeness and degree; given that they count all possible pathways. As a result, information indices can be more sensitive to changes than other indices.

\section{CONCLUSION}

This study stresses the importance of information centrality for capturing and assessing the coordinative activity within a project network. The proposed approach was further investigated in an in-depth case study involving electronic information exchange between the firms, which were the main stakeholders in a wayfinding signage project for Istanbul Sabiha Gökçen International Airport. The data was derived from a precedent study (Dogan et al. 2013) for a broader examination of the comparative roles based on the centrality indices. The computational procedures are provided with theoretical bases and methods. The study was carried out in two consecutive phases regarding correlations: the centrality indices associated with the firms' coordination scores were thoroughly investigated and the centrality indices were analyzed with other centrality 
measures in order to illustrate the reliability dimensions which may indicate the potential coordination performance.

The study used the concept of information centrality in conjunction with other three standard centrality measures to examine a group of firms which full email information and basic centrality measures were available. The correlation between the network position and coordination performance was verified by adding a new measure, which is information centrality.

The empirical evidence from the case study shows that the coordination scores are highly correlated with centrality indices (Table 3). This study also validates the procedure suggested by Dogan et al. (2013) for measuring the coordinative activity through centrality indices. Findings from this study motivate that the thorough analyses using information centrality indices in communication networks may result more robust and illustrative findings for the project roles, coordination performance and information reliability. From a theoretical standpoint, the method for measuring information centrality proposed by Stephenson and Zelen (1989) makes use of all paths between nodes rather than geodesic paths. The calculations based on this framework can be easily adopted and organized for larger project networks. The only limitation for this study can be given as the network size.

It can be claimed that the approach presented in this paper associated with the proposed computational procedures has the application potentials for rapid analysis of complex project organizations, robustness of information exchange or specifically changes in project networks in the case of new potential network nodes added or subtracted (Stephenson and Zelen 1989). The proposed approach has advantages over complex and expensive computational methods and graphic techniques for analyzing project networks and organizations, in terms of simplicity and practicality.

\section{REFERENCES}

Ahuja, M. K., Galletta, D. F., and Carley, K. M. (2003). "Individual centrality and performance in virtual R\&D groups: An empirical study." Management Science, 49(1), 21-38.

Bavelas, A. (1950). "Communication patterns in task oriented groups." The Journal of the Acoustical Society of America, 22(6), 725-730.

Borgatti, S. P., Everett, M. G., and Freeman, L. C. (2002). UCINET 6 for windows: software for social network analysis, Analytic Technologies, Harvard, MA.

Dogan, S. Z., Gunhan, S., and Erbasaranoglu, B. (2012). "Coordination Process and Network Centrality in ISGI Airport's Wayfinding Project." Construction Research Congress 2012: Construction Challenges in a Flat World, pp. 708-717

Dogan, S. Z., Arditi, D., Gunhan, S., and Erbasaranoglu, B. (2013). "Assesing Coordination Performance Based on Centrality in an Email Communication Network." Journal of Management in Engineering, in press.

Erbasaranoglu, B. (2011). "Analyzing the communication and coordination processes in Sabiha Gökçen International Airport's new terminal building wayfinding project", M.S. thesis, Izmir Institute of Technology, Izmir, Turkey. 
Freeman, L. C. (1978/79). "Centrality in social networks conceptual clarification." Social networks, 1(3), 215-239.

Freeman, L. C., Roeder, D., and Mulholland, R. R. (1979/80). "Centrality in social networks: ii. experimental results." Social Networks, 2(2), 119-141.

Hollander, M., and D. A. Wolfe (1999). Nonparametric statistical methods, 2nd ed., WileyInterscience, Hoboken, NJ.

Hossain, L., Wu, A., and Chung, K. K. S. (2006). "Actor centrality correlates to project based coordination." Proceedings of the 2006 20th anniversary conference on CSCW'06 Computer supported cooperative work, Banff, Alberta, Canada, 363-372.

Hossain, L. (2009). "Communications and coordination in construction projects." Construction Management and Economics, 27(1), 25-39.

Klimt, B., and Yang, Y. (2004). "The enron corpus: A new dataset for email classification research. Proceedings of the 2004 15th European conference on Machine Learning, Pisa, Italy, 217-228.

Leavitt, H. J. (1951). "Some effects of certain communication patterns on group performance." The Journal of Abnormal and Social Psychology, 46(1), 3850 .

Pryke, S. D. (2004). "Analysing construction project coalitions: exploring the application of social network analysis." Construction Management and Economics, 22(8), 787-797.

Schilling, M. A., and Phelps, C. C. (2007). "Interfirm collaboration networks: The impact of large-scale network structure on firm innovation." Management Science, 53(7), 1113-1126.

Stephenson, K., and Zelen, M. (1989). "Rethinking centrality: Methods and examples." Social Networks, 11(1), 1-37.

Tallberg, C. (2004). "Testing centralization in random graphs." Social Networks, 26(3), 205-219.

Wasserman, S., and Faust, K. (1994). Social Network Analysis: Methods and Applications, Cambridge University Press, Cambridge, UK. 